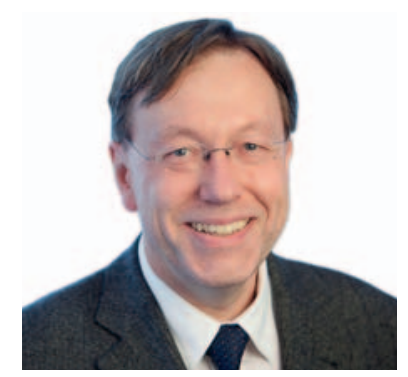

\title{
Lebensqualität als wichtiges Therapieziel im Gesamtbehandlungskonzept bei COPD
}

\author{
PD Dr. Helmut Frohnhofen
}

Medizinische Klinik 2, Alfried Krupp Krankenhaus Essen

\begin{abstract}
Corlateanu A, Botnaru V, Covantev S, Dumitru S, Siafakas N: Predicting health-related quality of life in patients with chronic obstructive pulmonary disease: the impact of age. Respiration 2016;92:229-234.
\end{abstract}

\section{Prädiktion der gesundheitsbezogenen Lebensqualität bei Patienten mit chronisch- obstruktiver Lungenerkrankung: Auswirkungen des Alters}

\section{Schlüsselwörter}

COPD · Lebensqualität · St. George's Respiratory Questionnaire · Klinischer COPD-Fragebogen

\section{Zusammenfassung}

Hintergrund: Die chronisch-obstruktive Lungenerkrankung (COPD) ist eine der Hauptursachen für Morbidität bei älteren Menschen. COPD geht mit einer beeinträchtigten gesundheitsbezogenen Lebensqualität (health-related quality of life, HRQoL) einher, allerdings sind die Faktoren dieser Beeinträchtigung bisher nicht hinreichend erforscht. Ein besseres Verständnis der maßgeblichen Faktoren für die HRQoL dürfte eine bessere Versorgung der betroffenen Patienten ermöglichen.

Ziele: Ziel dieser Studie war es, mögliche altersspezifische Unterschiede bei der HRQoL innerhalb einer Patientenpopulation mit ähnlichem Schweregrad der Obstruktion zu untersuchen.
Methoden: Insgesamt 180 konsekutive Patienten wurden in die Studie eingeschlossen. Wir analysierten Spirometriedaten, BODEIndex und dessen Komponenten sowie Charlson-Komorbiditätsindex. Die HRQoL wurde anhand zweier Fragebögen beurteilt: Clinical COPD Questionnaire (CCQ) und St. George's Respiratory Questionnaire (SGRQ).

Ergebnisse: Die Kohorte umfasste 93 «jüngere» Patienten (Durchschnittsalter 54,8 \pm 3,1 Jahre) und 87 Patienten höheren Alters (Durchschnittsalter 73,1 \pm 5,5 Jahre). In beiden Gruppen lag

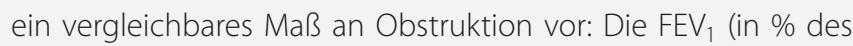
Sollwerts) betrug $39,9 \pm 13,2 \%$ in der älteren und $41,7 \pm 11,7 \%$ in der jüngeren Gruppe $(p>0,05)$. Eine schrittweise Regressionsanalyse mit Vorwärtsselektion ergab, dass der BODE-Index, der Charlson-Index und die Exazerbationsrate wichtige Prädiktoren für die Abnahme der HRQoL bei älteren COPD-Patienten darstellen; hierdurch lassen sich 29\% des gesamten SGRQ-Scores erklären. Bei den jüngeren COPD-Patienten betrug das Bestimmtheitsmaß $R^{2}$ 0,27, doch die Prädiktoren waren BODE-Index und Exazerbationsrate.

Schlussfolgerungen: BODE-Index, Charlson-Index und Exazerbationsrate erwiesen sich als Hauptdeterminanten der HRQoL bei COPD-Patienten höheren Alters, bei jüngeren COPD-Patienten waren es der BODE-Index und die Exazerbationsrate.

(c) 2017 S. Karger GmbH, Freiburg

\section{KARGER}

Fax +4976145207 14

information@karger.com www.karger.com
(๑) 2017 S. Karger GmbH, Freiburg 


\section{Transfer in die Praxis}

\section{Hintergrund}

Die Behandlung älterer Menschen stellt im Kontext von Multimorbidität eine große Herausforderung dar. Neben der kritischen Prüfung der Frage, ob überhaupt eine Behandlungsnotwendigkeit mit klar formulierten Therapiezielen gegeben ist, müssen auch die erforderliche Behandlungsintensität und -dauer klar festgelegt werden [1]. Wichtig ist auch die Berücksichtigung der wahrscheinlich noch verbleibenden Lebenszeit, auch wenn diese im Einzelfall nur schwer abgeschätzt werden kann. Gerade im höheren Lebensalter verliert bei Therapieentscheidungen die Prognose quoad vitam für den Patienten an Bedeutung, und Faktoren wie Funktionalität im Alltag, Selbstständigkeit, Kognition und Lebensqualität werden immer wichtiger [1].

Die Verbesserung der Lebensqualität ist ein zunehmend als wichtig anerkanntes und akzeptiertes Therapieziel. Die Operationalisierung des Konstruktes Lebensqualität allgemein ist nicht einfach [2]. Häufig wird daher die gesundheitsbezogene Lebensqualität als Outcomeparameter gewählt [2]. Für die chronisch-obstruktive Lungenerkrankung (COPD) sind mit dem Clinical COPD Questionnaire (CCQ) und dem St. George's Respiratory Questionnaire (SGRQ) spezifische und valide Instrumente für die Messung der gesundheitsbezogenen Lebensqualität verfügbar.

\section{Studienergebnisse}

In der Arbeit von Corlatenanu et al. wird nun die Frage des Einflusses des Lebensalters auf eventuelle Unterschiede in der gesundheitsbezogenen Lebensqualität bei Patienten mit COPD untersucht. Dazu wurden 180 konsekutive COPD-Patienten in einer monozentrischen Querschnittsuntersuchung nach dem Alter in zwei Gruppen eingeteilt. Als Altersgrenze wurde WHO-konform ein Lebensalter von 65 Jahren gewählt.

Die Gruppe der älteren Patienten unterschied sich signifikant von der Gruppe der Jüngeren im Umfang der Gesamtsymptomatik (BODE-Score), der Belastbarkeit (6-Minuten-Gehtest), der Dyspnoe (mMRC-Score) und in der Anzahl der zusätzlich vorliegenden Erkrankungen (Charlson-Index), nicht jedoch in den Parametern der Lungenfunktionsprüfung. Die Parameter der Lebensqualität waren für beide verwendeten Instrumente (CCQ und SGRQ) im Gesamtscore und in einzelnen Unterscores signifikant unterschiedlich.

In mehreren multiplen logistischen Regressionen waren bei jüngeren und älteren Patienten die Gesamtsymptomatik (BODEScore) und die Anzahl der jährlichen Exazerbationen signifikant mit der gesundheitsbezogenen Lebensqualität im CCQ und im SGRQ assoziiert. Zusätzlich fand sich bei den älteren Patienten eine signifikante Assoziation zwischen der gesundheitsbezogenen Lebensqualität und den bestehenden Komorbiditäten (Charlson-Index)

\section{Kritik und Fazit für die Praxis}

Das Ergebnis dieser Studie ist nicht überraschend. Gesamtsymptomatik und Exazerbationen sind bei Patienten mit COPD in allen Altersgruppen Determinanten der Lebensqualität. Zusätzlich zeigt diese Studie auch die Relevanz von Komorbiditäten für die gesundheitsbezogene Lebensqualität.

Die Studie umfasst 180 Patienten und ist damit relativ klein. Daher können wichtige Fragen, die sich aus diesen ersten Ergebnissen ergeben, naturgemäß nicht beantwortet werden. So wäre die Frage zu klären, welche Teilkomponenten der Lebensqualität (Aktivität, Funktionalität, Kognition) konkret mit dem Schweregrad einer COPD und/oder spezifischen Komorbiditäten (z. B. Herzinsuffizienz, Krebserkrankung, Diabetes mellitus) zusammenhängen. Der Charlson-Index subsumiert ja eine vorgegebene Anzahl von Komorbiditäten in einem Score, ohne dass einzelne Komorbiditäten berücksichtigt werden können. So werden z.B. Herzinsuffizienz, Demenz, Schlaganfall oder Diabetes mellitus mit jeweils einem Punkt bewertet, obwohl diese Erkrankungen ganz unterschiedlich die Lebensqualität beeinflussen können. Aus solchen Erkenntnissen ließen sich dann individualisiert spezifischere Therapiekonzepte erstellen.

Es wäre auch wichtig, die Effekte einer schon verordneten Therapie zu berücksichtigen. Konkret muss die Frage nach zusätzlich bestehenden Schmerzen, Frakturen im Rahmen einer Osteoporose und der Umfang einer verabreichten Schmerztherapie analysiert werden. Diese Rohdaten dürften vorliegen.

Dennoch ist diese Studie wichtig, da sie Assoziationen zwischen der Lebensqualität und Komponenten der COPD aufzeigt und Komorbiditäten als relevante Einflussfaktoren bei älteren Patienten identifiziert. Für den klinischen Alltag bedeutet dies, auch die Komponente Lebensqualität als wichtiges Therapieziel mit in ein Gesamtbehandlungskonzept aufzunehmen. Durch weitere Studien muss künftig geklärt werden, ob sich durch eine individualisierte Therapie auch die Lebensqualität bei Patienten mit COPD verbessern lässt.

\section{Disclosure Statement}

Hiermit erkläre ich, dass keine Interessenskonflikte in Bezug auf den vorliegenden Kommentar bestehen.

\section{Literatur}

1 Holmes HM, Hayley DC, Alexander GC, Sachs GA: Reconsidering medication appropriateness for patients late in life. Arch Int Med 2006;166: 605-609.

2 Conrad I, Matschinger H, Kilian R, Riedel-Heller S. WHOQOL-OLD und WHOQOL-BREF: Handbuch für die deutschsprachigen Versionen der WHOInstrumente zur Erfassung der Lebensqualität im Alter. Göttingen, Hogrefe, 2016.

Kontaktadresse: PD Dr. Helmut Frohnhofen, Medizinische Klinik 2, Alfried Krupp Krankenhaus Essen, Alfried-Krupp-Straße 21, 45131 Essen, Deutschland, helmut.frohnhofen@krupp-krankenhaus.de 